\title{
BMJ Open PCASTt/SPCG-17-a randomised trial of active surveillance in prostate cancer: rationale and design
}

Mats Steinholtz Ahlberg, ${ }^{1}$ Hans-Olov Adami, ${ }^{2,3}$ Kerri Beckmann, ${ }^{4,5}$ Helena Bertilsson, ${ }^{6,7}$ Ola Bratt, ${ }^{8}$ Declan Cahill, ${ }^{9}$ Lars Egevad, ${ }^{10}$ Hans Garmo, ${ }^{11,12}$ Lars Holmberg, ${ }^{12,13}$ Eva Johansson, ${ }^{1}$ Antti Rannikko, ${ }^{14}$ Mieke Van Hemelrijck, ${ }^{15}$ Fredrik Jäderling, ${ }^{16,17}$ Cecilia Wassberg, ${ }^{16}$ Ulrika W N Åberg, ${ }^{1}$ Anna Bill-Axelson ${ }^{1}$

To cite: Ahlberg MS, Adami H-0, Beckmann K, et al. PCASTt/ SPCG-17-a randomised trial of active surveillance in prostate cancer: rationale and design. BMJ Open 2019;9:e027860. doi:10.1136/ bmjopen-2018-027860

- Prepublication history for this paper is available online. To view these files, please visit the journal online (http://dx.doi org/10.1136/bmjopen-2018027860).

Received 19 November 2018 Revised 22 June 2019 Accepted 04 July 2019
Check for updates

(C) Author(s) (or their employer(s)) 2019. Re-use permitted under CC BY-NC. No commercial re-use. See rights and permissions. Published by BMJ.

For numbered affiliations see end of article.

Correspondence to Dr Mats Steinholtz Ahlberg; mats.ahlberg@surgsci.uu.se

\section{ABSTRACT}

Introduction Overtreatment of localised prostate cancer is substantial despite increased use of active surveillance. No randomised trials help define how to monitor patients or when to initiate treatment with curative intent. Methods and analysis A randomised, multicentre, intervention trial designed to evaluate the safety of an MRI-based active surveillance protocol, with standardised triggers for repeated biopsies and radical treatment. The aim is to reduce overtreatment of prostate cancer. 2000 men will be randomly allocated to either surveillance according to current practice or to standardised triggers at centres in Sweden, Norway, Finland and the UK. Men diagnosed in the past 12 months with prostate cancer, $\leq \mathrm{T} 2 \mathrm{a}$, prostate-specific antigen (PSA) $<15 \mathrm{ng} / \mathrm{mL}$, PSA density $\leq 0.2 \mathrm{ng} / \mathrm{mL} / \mathrm{cc}$, any International Society of Urological Pathology (ISUP) grade 1 are eligible. Men with ISUP grade 2 in $<30 \%$ of cores on systematic biopsy and $<10 \mathrm{~mm}$ cancer in one core on systematic or targeted biopsy are also eligible. Men diagnosed on systematic biopsy should have an MRI and targeted biopsies against Prostate Imaging and Reporting Data System V.2 3-5 lesions before inclusion. Identical followup in the two study arms: biannual PSA testing, yearly clinical examination and MRI every second year. In the experimental arm, standardised triggers based on MRI and PSA density elicit repeated biopsies. MRI and histopathological progression trigger radical treatment. Primary outcome measure is progression-free survival. Secondary outcome measures are cumulative incidence of metastatic disease, treatments with curative intent, pT3-4 at radical prostatectomy, switch to watchful waiting, prostate cancer mortality and quality of life. Inclusion started in October 2016 and in October 2018; 275 patients have been enrolled.

Ethics and dissemination Ethical approval was obtained in each participating country. Results for the primary and secondary outcome measures will be submitted for publication in peer-reviewed journals.

Trial registration number NCT02914873.

\section{INTRODUCTION}

Following the introduction of prostate-specific antigen (PSA) testing in the late 1980s, the incidence of prostate cancer increased

\section{Strengths and limitations of this study}

- The randomised design reduces chances of observed outcome being influenced by confounding factors.

- The trial size will allow quantifying clinically relevant end points with reasonable statistical precision.

- International multicentre study making results more generalisabe.

- Limitations include long follow-up that has to be undertaken to assess tumour progression.

dramatically in many countries. ${ }^{1}$ Widespread PSA testing led to a downward stage migration, with a growing proportion of small and well-differentiated cancers with low malignant potential even if left untreated. ${ }^{2}$

Although overdiagnosis of prostate cancers has been documented on a group level, ${ }^{2}$ currently it is not possible to know if an individual man with prostate cancer will experience progression to lethal disease or not. As a consequence, many men unnecessarily undergo radical treatment. To reduce overtreatment and its side effects, without jeopardising the potential benefit of radical treatment, active surveillance with selective, delayed therapy with curative intent was developed in the late 1990s. ${ }^{3}$

In active surveillance, treatment with curative intent is initiated when and if investigations indicate progressive cancer. In watchful waiting only palliative treatment is initiated at symptoms. Several national guidelines recommend active surveillance for most low-risk cancers and selected favourable intermediate-risk cancers. ${ }^{4}$ Different criteria are used to trigger radical treatment, but many patients are treated with curative intent without objective signs of disease progression. ${ }^{5}$ Although multiple active surveillance cohorts show low rates of 
disease progression, ${ }^{6}$ no randomised trials help define which patients are suitable for active surveillance, how to monitor them or when to initiate treatment with curative intent. To fill some of these evidence gaps and reduce both overtreatment and undertreatment, the Scandinavian Prostate Cancer Group (SPCG) is promoting a multinational randomised trial, Prostate Cancer Active Surveillance Trigger trial (PCASTt/SPCG-17), in which standardised triggers for repeat biopsies and initiation of treatment with curative intent, is compared with current clinical practice for active surveillance of low-risk and favourable intermediate-risk prostate cancer.

\section{Areas of uncertainty}

Active surveillance or immediate treatment with curative intent

None of three randomised trials has shown substantial survival benefit of radical treatment compared with watchful waiting or active monitoring in men with low-risk prostate cancer. ${ }^{7-9}$ The SPCG-4 trial began in 1989, that is, before the PSA era, and included primarily men with palpable tumours. In this trial, radical prostatectomy resulted in a $3.8 \%$ (95\% CI -4.6 to 12.2$)$ lower prostate cancer mortality in men with low-risk cancer, compared with watchful waiting, after 18 years of follow-up. ${ }^{7}$ In the PIVOT trial (Prostate Cancer Intervention Versus Observation Trial) including predominantly men with PSA detected localised cancer, there was a $4 \%$ absolute reduction of prostate cancer-specific mortality ( $95 \%$ CI -0.2 to 8.3) after radical prostatectomy, compared with observation, after nearly 20 years of follow-up. Events were few in the subgroup analysis, ensuing low statistical precision. ${ }^{8}$ In ProtecT, only men with PSA-detected tumours were included. After 10 years of follow-up, prostate cancer-specific survival was similar in the three treatment groups: 98.8\% (95\% CI 98.4 to 99.5$)$ after initial active monitoring, $99.0 \%$ (95\% CI 97.2 to 99.6 ) in men allocated to radical prostatectomy and $99.6 \%$ (95\% CI 98.4 to 99.9$)$ in men allocated to radiotherapy, but progression to metastatic disease was less common after treatment with curative intent. $^{9}$

\section{Criteria for active surveillance}

Most published active surveillance protocols include men with low-risk disease (International Society of Urological Pathology (ISUP) grade 1 (Gleason score 3+3=6), T1c-T2a and PSA $<10 \mathrm{ng} / \mathrm{mL}$ ), but some include intermediate-risk disease (ISUP grade 2 (Gleason score $3+4=7$ ), T1c-T2 and PSA $10-20 \mathrm{ng} / \mathrm{mL}) .{ }^{6}$ ProtecT, the only randomised trial addressing the question of active monitoring versus curative treatment, included all localised risk groups but predominantly low risk. They found a higher risk of progression to metastases with active monitoring compared with radical treatment, after 10 years. ${ }^{9}$ However, active monitoring in their protocol consisted of repeated PSA testing but apart from that was not specifically regulated.
Follow-up during active surveillance

Active surveillance protocols differ, but they typically include repeated digital rectal examinations, PSA testing and systematic, transrectal biopsies. Interpretation of digital rectal examination is subjective and cannot detect tumours in the anterior part of the prostate, which limit its usefulness. ${ }^{10}$ PSA values fluctuate over time, ${ }^{11}$ and raising values may reflect inflammation or progression of benign hyperplasia, rather than tumour progression. Systematic biopsies can easily miss small multifocal cancers and large tumours in the anterior prostate. Histopathological evaluation of the specimen shows considerable interindividual variation between pathologists. $^{12}$

\section{Repeated biopsy and conversion to radical treatment}

In Sweden, $30 \%-40 \%$ of men managed by active surveillance receive treatment with curative intent within 5 years after diagnosis. ${ }^{13}$ In a nationwide study, active surveillance was discontinued because of 'patient preference' in 20\%, by PSA progression in $52 \%$ and by biopsy progression in $24 \%$ of the men. ${ }^{13}$ In the PRIAS study (Prostate Cancer Research International Active Surveillance), about half of the men switched to curative treatment within 2.3 years. ${ }^{14}$ Worry about whether the patient has an undetected highrisk cancer, without objective signs of progression or high-risk cancer, may entail unnecessary repeated biopsies and treatment to accommodate the clinicians' and the patients' concerns. Conversely, digital rectal examination, PSA and systematic biopsies have low sensitivity to detect high-grade cancer. ${ }^{10-12}$ Hence, we do not know when repeated biopsies are required and when radical treatment is beneficial.

\section{Magnetic resonance imaging}

The use of MRI in the assessment of prostate cancer has increased. There is high level evidence that MRI has the ability to detect prostate cancer, ${ }^{15}{ }^{16}$ but there is insufficient evidence on the benefit of repeated MRI during active surveillance. ${ }^{17}$ According to the PROMIS study (Prostate MR Imaging Study), MRI may reduce the proportion of men undergoing a prostate biopsy by $25 \%$, and the proportion of men diagnosed with clinically insignificant cancer by $5 \%$, at the cost of delaying the diagnosis of a clinically significant cancer (defined as presence of any ISUP grade $\geq 3$ (Gleason score $4+3=7$ ) or a maximum cancer core length involvement of $6 \mathrm{~mm}$ or more in any location) in $3 \%$ of the men. ${ }^{18}$ The negative predictive value of an unsuspicious MRI finding is high, and even higher when PSA density is low. ${ }^{19}$

The randomised PRECISION (Prostate Evaluation for Clinically Important Disease: Sampling Using Image Guidance or Not?) trial indicates that MRI with targeted biopsies has higher detection rate than systematic biopsies for ISUP grade $\geq 2$ prostate cancer and reduces the detection of ISUP grade 1 cancer in biopsy-naive patients with clinical suspicion of prostate cancer. ${ }^{20}$ In a retrospective review of repeated biopsies during active surveillance, MRI with targeted biopsies nearly doubled the detection 
of pathological progression compared with systematic biopsies. ${ }^{21}$ This is however questioned by a recent prospective trial that showed no increase in upgrading with additional targeted biopsies versus systematic biopsies alone. ${ }^{22}$

\section{Active surveillance in intermediate-risk prostate cancer}

The risk of cancer progression during active surveillance of patients with low-risk prostate cancer is low, but varies between studies, probably because of different inclusion criteria and indications for therapeutic intervention. ${ }^{6}$ Active surveillance of intermediate-risk prostate cancer is debated, but it is supported by some data. ${ }^{23}$ The Sunnybrook cohort-including men with low-risk and favourable intermediate-risk prostate cancer-showed a $2.8 \%$ progression to metastatic disease and $1.5 \%$ prostate cancer-specific mortality within 15 years, ${ }^{24}$ with a more favourable outcome for men with low-risk cancer. ${ }^{25}$ Eligibility was not influenced by PSA density or number of positive cores, and MRI was not used. Based on findings that MRI with targeted biopsies has a higher detection rate than standard biopsies for ISUP grade $\geq 2$ prostate cancer, ${ }^{20}$ one must assume that many intermediate-risk and high-risk tumours were undetected in this cohort. Despite this, the long-term cancer-specific survival was high.

\section{The PCASTt/ SPCG-17 trial}

Study design

The PCASTt/SPCG-17 is a multinational randomised trial comparing active surveillance using standardised triggers for repeated biopsy and radical treatment with current practice. The hypothesis is that standardised triggers will reduce overtreatment and adverse events and improve quality of life, without increasing disease progression or prostate cancer mortality.

\section{Outcome measures}

The primary end point is progression-free survival. Progression is defined as biochemical recurrence after treatment with curative intent or start of androgen deprivation therapy in previously untreated men. Following radical prostatectomy, biochemical recurrence is defined as two consecutive rising PSA values $>0.2 \mathrm{ng} / \mathrm{mL}$. After primary radiation therapy and radiation therapy with androgen deprivation therapy, the definition of biochemical recurrence is any PSA increase $2 \mathrm{ng} / \mathrm{mL}$ higher than the PSA nadir value, regardless of the serum concentration of the nadir. ${ }^{26}$ Secondary end points are the cumulative incidence of pT3 tumours, distant metastasis, treatment with curative intent and switch to watchful waiting. Prostate cancer death is the final end point at 10 years. Quality-of-life end points will be analysed separately.

\section{Participants and participating centres}

Eligible for inclusion are men with histopathological low-risk or favourable intermediate-risk adenocarcinoma of the prostate diagnosed within 12 months, who have not received any treatment and have at least 10 years' expected lifetime. All men primarily diagnosed with

\section{Box 1 Inclusion criteria}

- Adenocarcinoma of the prostate diagnosed within the past 12 months.

> Tumour stage $\leq \mathrm{T} 2 \mathrm{a}, \mathrm{NX}, \mathrm{M} 0$.

- PSA $<15 \mathrm{ng} / \mathrm{mL}$ and PSA density $\leq 0.2 \mathrm{ng} / \mathrm{mL} / \mathrm{cc}$.

- Systematic biopsies with $\geq 10$ cores (optional, if the diagnosis is based on MRI with targeted biopsies).

- MRI with targeted biopsies towards PI-RADS 3, 4 and 5 (according to PI-RADS V.2).

- ISUP grade 1 (any number of cores, any involvement).

- ISUP grade 2 in $<3$ cores (or $<30 \%$ of cores if $>10$ systematic cores were taken) and $<10 \mathrm{~mm}$ cancer in one core (systematic or targeted).

- Life expectancy $\geq 10$ years (no upper age limit).

- Candidate for curative treatment (surgery or radiotherapy), if progression occurs.

- Signed written informed consent.

ISUP, International Society of Urological Pathology; PI-RADS, Prostate Imaging Reporting and Data System; PSA, prostate-specific antigen.

prostate cancer from systematic biopsies should undergo MRI with biopsies targeted towards Prostate Imaging and Reporting Data System (PI-RADS) 3-5 lesions before inclusion. For men primarily diagnosed with prostate cancer following MRI with targeted biopsies, subsequent systematic biopsies are optional. Inclusion criteria are listed in box 1. Centres in Sweden, Finland, Norway and the UK will participate. Demands on participating centres are listed in box 2.

PCASTt/SPCG-17 aims to randomise 2000 patients in 4 years, which will give an $85 \%$ power to detect a 1.3 percentage points progression-free survival difference between the study arms (two-sided $\alpha=0.05$ ) under the assumption that $90 \%$ of the patients are managed per protocol according to randomisation. The progression-free survival in the current practice group 5 years after randomisation is assumed to be $98 \%$, based on previous studies. ${ }^{24}$

Patient-reported outcome measures and follow-up

At inclusion and every second year during follow-up participants are requested to complete a study-specific

\section{Box 2 Demands on participating centres}

- Access to prostate MRI expertise. If the local competence is uncertain, the national PI will organise external expertise for MRI evaluation.

- The MRI should follow European Society of Urogenital Radiology guidelines and include:

- T1-weighted and T2-weighted images;

- Diffusion-weighted images including apparent diffusion coefficient;

- Dynamic contrast-enhanced imaging and magnetic resonance spectroscopy imaging are optional.

- The MR images should be reported according to PI-RADS V.2.

PI-RADS, Prostate Imaging Reporting and Data System. 
- Clinical check-up \&

PSA

-MRI+/-TB Inclusion

$\bullet+/-\mathrm{SB}$

-QoL questionnaire
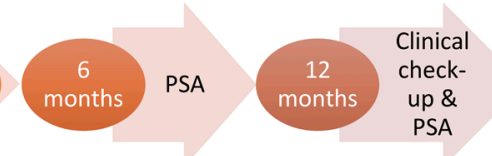

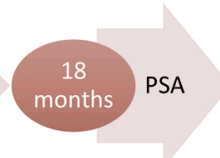

- Clinical check up \&PSA

-MRI

-QoL

questionnaire

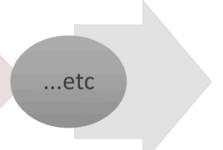

Figure 1 Basic follow-up. PSA, prostate-specific antigen; QoL, quality of life; SB, systematic biopsies; TB, targeted biopsies.

quality-of-life questionnaire including the Expanded Prostate cancer Index Composite $-26,{ }^{27}$ for separate quality-of-life analysis.

Basic follow-up is identical in both arms, with biannual PSA testing, annual clinical examination and MRI scan every second year (figure 1). In the current practice arm, additional investigations are optional and it is up to the urologist to decide when it is time to repeat biopsies and initiate treatment with curative intent. In the experimental arm, follow-up is according to schedule, biopsies are only taken if the standardised triggers are reached (table 1) and treatment with curative intent is only recommended if standardised triggers for radical treatment are reached (table 2). At every follow-up, the patient is categorised as having no distant metastasis, suspected distant metastasis (according to PSA-level and/or symptoms) or verified distant metastasis (imaging or histopathology/ cytology).

Follow-up continues according to the protocol until initiation of treatment with curative intent, detection of metastasis, switch from active surveillance to watchful waiting or death of any cause. For men who discontinue active surveillance, the follow-up and management continue according to standard clinical practice but with annual reporting in the study. In patients lost to follow-up, end points will be assessed through available registers in the participating countries.

\section{Patient and public involvement}

Patient experiences and priorities is important knowledge that may influence the definition of research questions. Patients were however not directly involved in designing this study, defining outcome measures, the recruitment to and conduct of the study. A summary of results will be available for all participants and will also be presented to patient organisations and the public.

\section{Experience from first 2 years of inclusion}

The first patient was included in October 2016 at Uppsala University Hospital, Sweden. In 2017, six additional Swedish centres began enrolment and three Norwegian centres started enrolling patients in 2018. Centres in Finland and the UK aim to start including patients later in 2018. Characteristics of the first 275 randomised patients are displayed in table 3. Patients are stratified based on participating centre and Gleason score.

\section{Ethics and dissemination}

The first analysis of primary and secondary end points will take place 1 year after all men are included (and then every third year) and will be published in peer-reviewed journal.

\section{DISCUSSION}

The increasing use of active surveillance has reduced overtreatment of prostate cancer to some extent, but still

\section{Table 1 Triggers for re-biopsies}

\begin{tabular}{|c|c|c|}
\hline Arm 1 & Arm 2 & \\
\hline \multirow{2}{*}{$\begin{array}{l}\text { According to current practice } \\
\text { (the urologists' judgement) }\end{array}$} & 1 & PSA density $>0.2 \mathrm{ng} / \mathrm{mL} / \mathrm{cc}$ (systematic biopsies) \\
\hline & III & $\begin{array}{l}\text { MRI progression in men with ISUP grade } 2 \text { cancer (targeted biopsies) } \\
\geq 5 \mathrm{~mm} \text { or more increase in size in any dimension of a measurable lesion (a } \\
\text { measurable lesion is defined as } \geq 6 \mathrm{~mm} \text { in longest diameter in any dimension in } \\
\text { best-depicted MR sequence) } \\
\text { A new lesion with PI-RADS } 3-5\end{array}$ \\
\hline
\end{tabular}

ISUP, International Society of Urological Pathology; PI-RADS, Prostate Imaging Reporting and Data System; PSA, prostate-specific antigen. 
Table 2 Triggers for radical treatment

\begin{tabular}{lll}
\hline Arm 1 & Arm 2 & \\
\hline $\begin{array}{l}\text { According to current } \\
\text { practice } \\
\text { (the urologist's judgement) }\end{array}$ & MRI progression in lesions with confirmed Gleason pattern 4 \\
& Increase in PI-RADS score to 4 or 5 \\
& High suspicion of extracapsular extension or seminal vesicle invasion (level of \\
& suspicion to be 4 or 5 on the Likert scale) \\
& Pathological progression \\
& Gleason pattern 5 \\
& Primary Gleason pattern 4 in any core with $\geq 5$ mm cancer \\
& ISUP grade 2 in $\geq 3$ cores (or $\geq 30 \%$ of cores if $>10$ systematic cores), or $\geq 10$ mm \\
& cancer in one core (systematic or targeted) \\
\hline
\end{tabular}

ISUP, International Society of Urological Pathology; PI-RADS, Prostate Imaging Reporting and Data System.

a large number of men are overtreated. ${ }^{2}$ Clearly, more patients should start on active surveillance, and fewer patients on active surveillance should convert to radical treatment.

Over the last decades, there has been a Gleason score/ ISUP grade inflation, partly due to the 2005 revision of

\begin{tabular}{|c|c|c|}
\hline $\mathbf{n}$ & 139 (Arm 1) & 136 (Arm 2) \\
\hline Age (year, mean (SD)) & $62(6.2)$ & $64(6.2)$ \\
\hline PSA (ng/mL, mean, (SD)) & $5.1(2.3)$ & $5.8(2.3)$ \\
\hline PSA-D (ng/mL/cc, mean (SD)) & $0.11(0.04)$ & $0.12(0.04)$ \\
\hline \multicolumn{3}{|l|}{ Clinical tumour stage (n (\%)) } \\
\hline T1c & $109(78)$ & $115(85)$ \\
\hline T2a & $29(21)$ & $21(15)$ \\
\hline Unknown & $1(1)$ & 0 \\
\hline \multicolumn{3}{|l|}{ Comorbidity (n (\%)) } \\
\hline ASA 1 & $94(68)$ & $84(62)$ \\
\hline ASA 2 & $40(29)$ & $52(38)$ \\
\hline ASA 3 & $4(3)$ & 0 \\
\hline Unknown & $1(1)$ & 0 \\
\hline \multicolumn{3}{|l|}{ Family history of PCA (n (\%)) } \\
\hline Yes & $33(24)$ & $38(28)$ \\
\hline No & $102(73)$ & $95(70)$ \\
\hline Unknown & $4(3)$ & $3(2)$ \\
\hline \multicolumn{3}{|l|}{ MRI technique (n (\%)) } \\
\hline $1.5 \mathrm{~T}$ & $20(14)$ & $16(12)$ \\
\hline $3 \mathrm{~T}$ & $118(85)$ & $116(85)$ \\
\hline Unknown & $1(1)$ & $4(3)$ \\
\hline \multicolumn{3}{|l|}{ MRI findings ( $\mathrm{n}(\%)$ ) } \\
\hline PI-RADS 1-2 & $76(55)$ & $55(40)$ \\
\hline PI-RADS 3-5 & $63(45)$ & $81(60)$ \\
\hline
\end{tabular}

ASA, American Society of Anesthesiologists classification; PCA, prostate cancer; PI-RADS, Prostate Imaging Reporting and Data System; PSA, prostate-specific antigen; $n$, sample size. the Gleason pattern definitions. The revision entailed that many patterns previously designated Gleason pattern 3 are now reported as pattern 4 and that the Gleason score/ISUP grade on needle biopsies always includes the highest Gleason grade, even if it is just a minimal component. ${ }^{28}$ Because long-term outcomes of active surveillance and watchful waiting are chiefly based on studies undertaken before the 2005 revision, a substantial proportion of low-risk tumours in those studies would today be classified as intermediate risk. Despite this, they had excellent survival without treatment. These results and the changes in Gleason grading suggests that also men with favourable intermediate-risk prostate cancer should be offered active surveillance. This is supported by the fact that MRI with targeted biopsies detects favourable intermediate-risk tumours that standard biopsies do not detect. ${ }^{180}$ Hence, if all men with intermediate-risk prostate cancer undergo immediate radical treatment, overtreatment would increase.

In PCASTt/SPCG-17, follow-up during active surveillance is based on PSA testing and repeated MRI, not on repeated systematic biopsies. Prostate biopsies are uncomfortable and distressful. They cause bleeding, sometimes urinary retention and about $6 \%$ experience febrile infection and $1 \%$ develop sepsis. ${ }^{29}$ The incidence of serious infections is rising because of multidrug-resistant bacteria. ${ }^{30}$ It is therefore desirable to reduce the number of biopsies during active surveillance. In the experimental arm of PCASTt/SPCG-17, biopsies are only performed when triggered which will likely reduce the number of biopsies. MRI with targeted biopsies detect at least as many histopathological intermediate-risk tumours as standard biopsies in biopsy-naïve men, ${ }^{20}{ }^{21}$ suggesting that MRI with targeted biopsies is viable in a surveillance programme.

In PCASTt/SPCG-17, prespecified changes in MRI trigger targeted biopsies to assess histopathological progression. Since histopathological progression may occur without MRI changes, the protocol also stipulates systematic biopsies if PSA density increases above prespecified limits (table 1). Radical treatment is triggered by MRI findings suggesting progression of a known 
ISUP grade 2 tumour and by biopsies showing more than a defined upper limit of Gleason pattern 4 or any Gleason pattern 5 (table 2).

The strengths of PCASTt/SPCG-17 include the randomised design and a trial size that will allow quantifying clinically relevant end points with reasonable statistical precision. A data monitoring and safety committee will oversee patient safety and the trial's scientific integrity. The safety of the MRI-based follow-up will be regularly evaluated by comparing the outcome with a matched group of men managed by active surveillance in the Swedish SAMS study (Study of Active Monitoring in Sweden), who are on active surveillance based on systematic transrectal biopsies. ${ }^{31}$ During the long-term follow-up, new methods for monitoring and treatment might be introduced and applied in our patient cohort, obscuring the interpretation of the results. Furthermore, over time the triggers for repeated biopsies and treatment in the PCASTt/SPCG-17 trial's standard treatment arm might become more similar to the management of the men in the experimental group. Although this will affect the trial's ability to detect any difference between the two groups, the PCASTt/SPCG-17 experimental arm can still be used to assess the clinical safety and acceptability of a standardised protocol and provide prospective data on the performance of MRI as a monitoring tool-both aspects key to patient safety under active surveillance.

\section{CONCLUSION}

PCASTt/SPCG-17 is a randomised trial that evaluates the safety of an MRI-based active surveillance protocol, comparing standardised triggers for repeat biopsies and curative treatment. If the protocol proves to be as safe as current clinical practice, its implementation could lead to a reduction of the number of biopsies, reduce overtreatment of prostate cancer without compromising the outcome of the patients in terms of morbidity and mortality.

\footnotetext{
Author affiliations

${ }^{1}$ Uppsala Universitet Institutionen for Kirurgiska Vetenskaper, Uppsala, Sweden

${ }^{2}$ Karolinska Institutet Department of Medical Epidemiology and Biostatistics,

Stockholm, Sweden

${ }^{3}$ Department of Epidemiology, Harvard University T H Chan School of Public Health, Boston, Massachusetts, USA

${ }^{4}$ King's College London Translational Oncology and Urology Research, London, UK

${ }^{5}$ University of Southern Australia Centre for Population Health Research, Adelaide, South Australia, Australia

${ }^{6}$ Department of Urology, Sankt Olavs Hospital Universitetssykehuset i Trondheim, Trondheim, Norway

${ }^{7}$ Department of Cancer Research and Molecular Medicine, NTNU—Norwegian University of Science and Technology, Trondheim, Norway

${ }^{8}$ Department of Urology, Goteborgs universitet Sahlgrenska Akademin, Goteborg, Sweden

${ }^{9}$ The Royal Mardsen Hospital, London, London, UK

${ }^{10}$ Department of Pathology, Karolinska Universitetssjukhuset, Stockholm, Sweden

${ }^{11}$ School of Cancer \& Pharmaceutical Sciences, King's College London, London, UK

${ }^{12}$ Regional Cancer Centre Uppsala/Örebro, Uppsala, Sweden

${ }^{13}$ Division of Cancer Studies, King's College London School of Medicine, London, UK

${ }^{14}$ Helsinki University and Helsinki University Hospital, Helsinki, Finland
}

${ }^{15}$ Translational Oncology \& Urology Offices, Kings College London, London, UK ${ }^{16}$ Radiology Department, Karolinska Universitetssjukhuset, Stockholm, Sweden ${ }^{17}$ Karolinska Institutet Department of Molecular Medicine and Surgery, Stockholm, Sweden

Acknowledgements The authors would like to thank the following contributors to the study: Monica Andersson, Kerstin Almroth, Ann-Charlotte Borgefeldt, Britt-Inger Dahlin, Christina Danewid, Kristina From, Chatrine Hasselberg, Pernilla Helgesson, Helene Krook, Christofer Lagerros, Malin Lindell, Tuomas Mirtti, Annica Nilsson, Madeleine Nilsson, Hanna Nyberg, Merja Rignell, Inger Johanne Stokkan, Malin Ståhlgren, Øystein Størkersen and the SPCG-17 study group.

Collaborators SPCG-17 study group: Johan Stranne; Jonas Wallström; Pär DahIman; Bengt Friedrich; Conny Ström; Periklis Koumoutsakos; Annika Larsson; Mattias Tell; Magnus Alm; Jon Forsberg; Aus Saudi; Ove Andrén; Wolfgang Krauss; Hanna Vasarainen; Anu Kenttämies; Sverre Langørgen

Contributors All authors have made the following contributions to the work: substantial contributions to the conception or design of the work; or the acquisition, analysis or interpretation of data for the work. Drafting the work or revising it critically for important intellectual content. Final approval of the version to be published. Agreement to be accountable for all aspects of the work in ensuring that questions related to the accuracy or integrity of any part of the work are appropriately investigated and resolved.

Funding This study was supported by grants from the Swedish Cancer Society (2016/466 and 2014/1275), the Swedish Research Council (2016-00177 and 2016-01293), the Nordic Cancer Union and the Percy Falk Foundation to Dr BillAxelson and the Karolinska Institutet Distinguished Professor Award to Dr HansOlov Adami (2368/10-221).

Competing interests None declared.

\section{Patient consent for publication Not required.}

Ethics approval The study has ethical approval from Sweden (the Regional Ethical Review Board in Uppsala), Norway (REK Midt-Regional Ethics Committee Central), Finland (Ethics Committee of Surgery, Helsinki University Hospital) and UK (East of England-Essex Research Ethics Committee).

Provenance and peer review Not commissioned; externally peer reviewed.

Data availability statement No data are available.

Open access This is an open access article distributed in accordance with the Creative Commons Attribution Non Commercial (CC BY-NC 4.0) license, which permits others to distribute, remix, adapt, build upon this work non-commercially, and license their derivative works on different terms, provided the original work is properly cited, appropriate credit is given, any changes made indicated, and the use is non-commercial. See: http://creativecommons.org/licenses/by-nc/4.0/.

\section{REFERENCES}

1. Ferlay JSI, Ervik M, Dikshit R, et al. V1.0, cancer incidence and mortality worldwide: IARC CancerBase No. 11. 2013. Lyon, France: International Agency for Research on Cancer, 2012.

2. Klotz L. Prostate cancer overdiagnosis and overtreatment. Curr Opin Endocrinol Diabetes Obes 2013;20:204-9.

3. Klotz LH. Active surveillance for good risk prostate cancer: rationale, method, and results. Can J Urol 2005;12(Suppl 2):21-4.

4. Bruinsma SM, Bangma CH, Carroll PR, et al. Active surveillance for prostate cancer: a narrative review of clinical guidelines. Nat Rev Urol 2016;13:151-67.

5. Simpkin AJ, Tilling K, Martin RM, et al. Systematic review and meta-analysis of factors determining change to radical treatment in active surveillance for localized prostate cancer. Eur Urol 2015;67:993-1005.

6. Dall'Era MA, Albertsen PC, Bangma C, et al. Active surveillance for prostate cancer: a systematic review of the literature. Eur Urol 2012;62:976-83.

7. Bill-Axelson A, Holmberg L, Garmo H, et al. Radical prostatectomy or watchful waiting in early prostate cancer. $N$ Engl J Med 2014;370:932-42.

8. Wilt TJ, Jones KM, Barry MJ, et al. Follow-Up of prostatectomy versus observation for early prostate cancer. $N$ Engl J Med 2017;377:132-42.

9. Hamdy FC, Donovan JL, Lane JA, et al. 10-Year outcomes after monitoring, surgery, or radiotherapy for localized prostate cancer. $N$ Engl J Med 2016;375:1415-24. 
10. Naji L, Randhawa H, Sohani Z, et al. Digital rectal examination for prostate cancer screening in primary care: a systematic review and meta-analysis. Ann Fam Med 2018;16:149-54.

11. Eastham JA, Riedel E, Scardino PT, et al. Variation of serum prostatespecific antigen levels: an evaluation of year-to-year fluctuations. JAMA 2003;289:2695-700.

12. Berney DM, Algaba F, Camparo P, et al. The reasons behind variation in Gleason grading of prostatic biopsies: areas of agreement and misconception among 266 European pathologists. Histopathology 2014;64:405-11.

13. Loeb S, Folkvaljon Y, Makarov DV, et al. Five-Year nationwide follow-up study of active surveillance for prostate cancer. Eur Urol 2015;67:233-8.

14. Drost F-JH, Rannikko A, Valdagni R, et al. Can active surveillance really reduce the harms of overdiagnosing prostate cancer? A reflection of real life clinical practice in the PRIAS study. Trans/ Androl Urol 2018;7:98-105.

15. Fütterer JJ, Briganti A, De Visschere $P$, et al. Can clinically significant prostate cancer be detected with multiparametric magnetic resonance imaging? A systematic review of the literature. Eur Urol 2015;68:1045-53.

16. Weinreb JC, Barentsz JO, Choyke PL, et al. PI-RADS Prostate Imaging - Reporting and Data System: 2015, Version 2. Eur Urol 2016;69:16-40.

17. Scarpato KR, Barocas DA. Use of $m p M R I$ in active surveillance for localized prostate cancer. Urol Oncol 2016;34:320-5.

18. Ahmed HU, El-Shater Bosaily A, Brown LC, et al. Diagnostic accuracy of multi-parametric MRI and TRUS biopsy in prostate cancer (PROMIS): a paired validating confirmatory study. Lancet 2017;389:815-22.

19. Distler FA, Radtke JP, Bonekamp D, et al. The value of PSA density in combination with PI-RADS ${ }^{\mathrm{TM}}$ for the accuracy of prostate cancer prediction. J Urol 2017;198:575-82.

20. Kasivisvanathan V, Rannikko AS, Borghi M, et al. MRI-Targeted or standard biopsy for prostate-cancer diagnosis. N Engl J Med Overseas Ed 2018;378:1767-77.
21. Frye TP, George AK, Kilchevsky A, et al. Magnetic resonance Imaging-Transrectal ultrasound guided fusion biopsy to detect progression in patients with existing lesions on active surveillance for low and intermediate risk prostate cancer. J Urol 2017;197:640-6.

22. Klotz L, Loblaw $A$, Sugar $L$, et al. Active surveillance magnetic resonance imaging study (ASIST): results of a randomized multicenter prospective trial. Eur Urol 2019;75:300-309.

23. Klotz L. Active surveillance for intermediate risk prostate cancer. Curr Urol Rep 2017;18:80.

24. Klotz L, Vesprini D, Sethukavalan P, et al. Long-Term follow-up of a large active surveillance cohort of patients with prostate cancer. $J$ Clin Oncol 2015;33:272-7.

25. Musunuru HB, Yamamoto T, Klotz L, et al. Active surveillance for intermediate risk prostate cancer: survival outcomes in the Sunnybrook experience. J Urol 2016;196:1651-8.

26. Cornford P, Bellmunt J, Bolla M, et al. EAU-ESTRO-SIOG guidelines on prostate cancer. Part II: treatment of relapsing, metastatic, and castration-resistant prostate cancer. Eur Urol 2017;71:630-42.

27. Szymanski KM, Wei JT, Dunn RL, et al. Development and validation of an abbreviated version of the expanded prostate cancer index composite instrument for measuring health-related quality of life among prostate cancer survivors. Urology 2010;76:1245-50.

28. Danneman D, Drevin L, Robinson D, et al. Gleason inflation 1998 2011: a registry study of 97,168 men. BJU Int 2015;115:248-55.

29. Lundström K-J, Drevin L, Carlsson S, et al. Nationwide population based study of infections after transrectal ultrasound guided prostate biopsy. J Urol 2014;192:1116-22.

30. Zowawi HM, Harris PNA, Roberts MJ, et al. The emerging threat of multidrug-resistant gram-negative bacteria in urology. Nat Rev Urol 2015;12:570-84

31. Bratt O, Carlsson S, Holmberg E, et al. The study of active monitoring in Sweden (SAMS): a randomized study comparing two different follow-up schedules for active surveillance of low-risk prostate cancer. Scand J Urol 2013;47:347-55. 